\title{
Perceptions of the effects on professional role boundaries when introducing a nurse practitioner into general practice
}

Shirley Reveley Department of Nursing Studies, St Martin's College Education Centre, Cumberland Infirmary, Carlisle, UK

\begin{abstract}
This paper is concerned with how roles within one group general practice are perceived to have been affected by the introduction of the nurse practitioner into the primary health care team (PHCT) for a 2-year pilot period. The data presented are from data elicited during a single round of interviews with patients $(n=30)$ and two rounds of interviews with all medical and nursing staff within the primary health care team on two occasions (40 interviews). The patient interviews were undertaken in the first year of the study, and interviews with staff were undertaken within 6 months of the commencement of the study and during the final 6 months. The overall aim was to evaluate the feasibility of the role and its possible future application to other primary health care settings. This paper will discuss the findings of that aspect of the study relating to the effects on role boundaries between doctors and nurses, and between nurse practitioner and nurses, midwives and health visitors in the practice as perceived by the patients, nurses and doctors involved. The nurse practitioner in this particular practice was a very experienced practice nurse with a wide range of knowledge and skills which she brought to the nurse practitioner role. This, together with well-organized teaching and supervision in the practice setting and a structured programme of formal education, resulted in a highly effective practitioner who was generally seen to have blurred the boundary lines between medical and nursing roles.
\end{abstract}

Key words: nurse practitioners; patient perceptions; primary health care team; professional perceptions; role boundaries

\section{Introduction}

Role boundaries between professionals within health care are currently being reshaped, and it has been argued that the nurse practitioner role has become a test bed for the carving out of new roles and territories (Reveley, 1999). This paper is concerned with one such role, namely that of the nurse practitioner. In particular, it is concerned with how roles within one group general practice are perceived to have been affected by the introduction of

Address for correspondence: Dr Shirley Reveley, Department of Nursing Studies, St Martin's College, Fusehill Street, Carlisle CA12HH, UK. Email: s.reveley@ucsm.ac.uk

(C)Arnold 2001 the nurse practitioner into the primary health care team (PHCT) for a 2-year pilot period. The overall aim was to evaluate the feasibility of the role and its possible future application to other primary health care settings. This paper will discuss the findings of that aspect of the study relating to the effects on role boundaries between doctors and nurses in the practice as perceived by the patients, nurses and doctors involved.

The nurse practitioner was new to the practice, although she had previously worked extensively in primary health care and was an experienced practice nurse with several qualifications to her credit. The pilot scheme necessitated her undertaking the BSc(Hons) Nurse Practitioner degree on a parttime basis and also receiving in-house training and 1463-4236(2001)PC053OA 
support from the GPs. The role she was to undertake was defined as 'triage nurse practitioner' (see Box 1 for an outline of the role).

Box 1 Role description for triage nurse in the case-study practice

Receiving patients with undiagnosed, undifferentiated problems who request a same-day appointment with a doctor

Making an assessment of their health needs based on nursing knowledge and skills, including physical examination where appropriate

Making decisions about management of the patient's problem, including self-care, referral to a GP, prescription of medicines, clinical investigations, referral to secondary services or other agencies, or discharge from the health care system

\section{Literature review}

The shifting of roles within health care has emerged against a backdrop of such factors as changes in the General Practitioner Contract, manpower shortages, shifts in the interface between primary and secondary care, increased incidence of day surgery and early discharge from hospital (Jenkins-Clarke et al., 1997). Many nurse practitioners in primary health care settings see patients on their own responsibility, make diagnostic and treatment decisions and consult GP colleagues or refer to other health care or social agencies as appropriate (Burke-Masters, 1986; Poulton, 1995; Kaufman, 1996). Nurse practitioners utilize a range of knowledge and skills, some of which were formerly in the medical domain. It has been argued that nurses operating in this way are no more than 'mini-doctors' (Pearson, 1996). The nurse practitioner role as doctor substitute causes concern that it may lead to an erosion of the very essence of nursing (Greenfield et al., 1987). There appears to be a division between those who see the role of the nurse practitioner as a substitute doctor
(Pearson, 1996) and those who see it as an advanced nursing role (Department of Health and Social Security, 1986; Cable, 1994; Emmerson, 1996). MacGuire (1980), in her work on extended and expanded nursing roles, described two models which underlie the literature.

Model A sees nursing and medicine as two distinct disciplines. Commentators are concerned about the possibility of nursing functions being lost from the new role in favour of medical tasks.

Model B states that there are many tasks to be carried out in order to maintain the health of communities and to care for patients. Who does what is immaterial provided that they are trained for the task, competent, acceptable to patients and achieve the same standards.

However, making such distinctions does nothing to promote nursing as a united profession. Within general medical practice, nurses have long been expanding and extending their role to the benefit of patients, not just doctors. Indeed, Emmerson (1996) argues that much of the nurse practitioner's role simply makes explicit what many nurses have known for years.

Although there is a growing body of literature on nurse practitioners in primary health care, little has been reported on the effects on established roles. The literature tends to report the confusion that abounds as to what the differences are between - for example, hospital nurse practitioners and clinical nurse specialists, and nurse practitioners and practice nurses in primary care settings (Dickson, 1996). Kaufman (1996) reported tension and conflict associated with developing the role in general medical practice, with colleagues being concerned about the implication that current practice was not good enough and that it leads to an erosion of nursing. Hupcey (1993), in a study of nurse practitioners in the USA, reported that one of the main barriers to the development of the role was the attitude of other nurses. She notes that:

it is remarkable that nurse practitioners still feel a resistance from staff nurses towards their role. After 30 years, nurse practitioners should be well accepted by other members of their own profession.

(Hupcey, 1993: 184)

Role boundary changes do not just affect professionals. Some commentators suggest that 
disturbing the established relationships between doctors and nurses also involves disturbing the relationship between doctors, nurses and patients (Stilwell, 1988). Witz (1994) suggests that the power relationship between doctors and patients is disturbed if nurses take on an enhanced nursing role in which the therapeutic relationship is between patient and nurse rather than between patient and doctor. This is in contrast to the extended nursing role in which nurses take on medical tasks but doctors are still in charge. Oakley (1984) suggests that nurses are more closely in tune with the self-stated needs of the patient than with the self-stated needs of the medical profession, and patients' perceptions of the status of nurses in relation to their own position may facilitate nurses' enquiries about patients' management of their own health. Molde and Diers (1985) suggest that nurse practitioners' style of consultation adopts a partnership stance with patients and is responsible for the high levels of care and acceptance that have been shown in many North American studies.

\section{The study}

Fieldwork was undertaken between November 1994 and July 1997, with the following aims:

1) to evaluate the effectiveness of the nurse practitioner triage role in one general practice;

2) to explore perceptions of both the primary health care team and patients in the practice population, with regard to the role of the nurse practitioner in general practice;

3) to investigate the extent to which the introduction of the nurse practitioner was perceived to affect traditional role boundaries between doctors and nurses;

4) to identify the professional activities of the nurse practitioner.

\section{Methodology}

The research utilized a case-study approach involving the gathering of quantitative and qualitative data. This was collected by means of two rounds of interviews with doctors and nurses $(n=40)$, one at the start of the study period and one at the end (see Box 2), a patient satisfaction survey consisting of a semi-structured questionnaire $(n=300)$ and interviews with patients $(n=30)$, two audits of the nurse practitioner's work, one at the beginning of the study period and one at the end, an audit of the nurse practitioner's prescribing patterns, a survey designed to compare the work of the nurse practitioner with that of the GPs, and a patient perception survey of their consultation with either the nurse practitioner or a GP $(n=60)$.

Box 2 Members of primary health care team interviewed (doctors and nurses either working within or attached to the group general practice, including the nurse practitioner herself $-100 \%$ sample).

Staff interviewed at the start of the pilot study At the commencement of the study, in November 1994, there were eight GP partners in the group practice, three practice nurses, 15 clerical/reception staff, five attached district nurses, three attached health visitors and three midwives serving a practice population of 14376 patients. Two partners were female, one of whom took a lead role in family planning services.

Staff interviewed at the end of the pilot study Three GPs had retired from the practice and two new GPs had been employed (one male and one female). In addition, the practice had achieved fundholding status and a practice manager had been appointed. Two health visitors had left the area and been replaced by two others, and the midwives had also changed. The staff interviewed were therefore not exactly the same as in the first-stage interviews. However, this reflects the dynamic nature of any organization and had to be worked with. All new staff agreed to take part in the study, and indeed were very interested in it. 


\section{Scope of the paper}

This paper is confined to a discussion of the perceptions of doctors, nurses, health visitors, midwives, the nurse practitioner herself and patients, with regard to the impact that the nurse practitioner role has had on established role boundaries within the primary health care team. The data presented were elicited during a single round of interviews with patients $(n=30)$ and two rounds of interviews with all medical and nursing staff within the primary health care team on two occasions (40 interviews). The patient interviews were undertaken in the first year of the study, and interviews with staff were conducted within 6 months of the commencement of the study and during the final 6 months.

The 30 patients who were interviewed were a subset of the 300 patients participating in the patient questionnaire survey who had indicated on the questionnaire that they would be willing to be interviewed (see Table 1). They were all patients who had requested same-day appointments, and it was the view of the GPs that the sample is a fair representation of patients across the practice who request same-day appointments for urgent problems. As there were changes to the membership of the primary health care team over the course of the project, Box 2 shows the composition of the primary health care team at each interview period.

\section{Findings}

The extent to which the introduction of the nurse practitioner was perceived to affect traditional role boundaries between doctors and nurses will be illustrated by means of extracts from the data.

Table 1 Characteristics of the patient interview sample

\begin{tabular}{lllllllll}
\hline & \multicolumn{5}{c}{ Age (years) } & Total \\
\cline { 2 - 6 } & $15-19$ & $20-29$ & $30-39$ & $40-49$ & $50-59$ & $\geqslant 60$ & \\
\hline Male & 0 & 1 & 2 & 1 & 1 & 0 & 5 \\
Female & 2 & 5 & 11 & 2 & 4 & 1 & 25 \\
Total & 2 & 6 & 13 & 3 & 5 & 1 & 30 \\
\hline
\end{tabular}

\section{Practice nurses}

Nurse practitioner and practice nurse roles were rarely seen to overlap. This was explained by practice nurses as being due to a well-defined division of labour operating among the practice nurse team which had not been destabilized by the introduction of the nurse practitioner. The organization of nursing work within the practice was explained as follows:

There is no effect on the practice nurse's role because clinics are well used and well established. These clinics are practice nurse led with a GP in attendance. Practice nurses in this practice are using different skills (e.g., health promotion, elderly screening). Nurses have individual skills between them; they ask each other.

(Practice Nurse 1)

The three practice nurses who were interviewed each had their own separate sphere of activity, but helped each other out at busy times and during holiday periods. Thus there was one nurse who dealt with treatment-room work, one who undertook screening of the elderly population and ran clinics for chronic disease management, and one who undertook health promotion work.

The nurse practitioner was perceived as operating in a sphere of activity that was closely aligned to the work of the GPs, and was concerned with 'urgent' patients, rather than undertaking a practice nurse role. There was also a perception that the nurse practitioner has different skills, as explained by one practice nurse: 'The nurse practitioner has extra training in diagnosis'. Another said: 'It makes no difference to my role. My work is health promotion; no emergency stuff'.

There was also a perception that the nurse practitioner actually reduced the work of the treatment room nurses:

We are not seeing as many patients each day since she came. I think it will cut down on the practice nurses' work because when doctors see patients they automatically send them to us, but the nurse practitioner might deal with them herself.

Nursing roles were often explained in terms of tasks, and this view was borne out by patients. When asked how they perceived the work of the nurse practitioner in relation to that of other nurses, 
it was practice nurses that patients used as a comparison. Patients saw clear differences between the roles of nurse practitioners and practice nurses. All of the respondents perceived practice nurses as being there for 'technical' procedures, and as having less time than the nurse practitioner. In the words of one respondent:

Practice nurses are there for routine tasks. Nurse practitioners are there for individual problems. I've been with the little one for his injections. That's their job, not to talk and find out. With the nurses you don't actually go with a problem, it's just sort of general health checks and blood tests whereas the nurse practitioner's there for individual problems. If you want stitches and that out you'd go to the nurse whereas the nurse practitioner is there to give you medicine if you need it. Just double check what the doctor's to sign for.

(Interview 8)

One woman thought that the nurse practitioner had a more specialized role:

I was aware of the difference because she was more knowledgeable ... I thought it was something like that carrying on from where the general nurse leaves off.

(Interview 5)

Another explained the difference as follows:

You see the other nurses for stitches and things, whereas the nurse practitioner to me was just like a doctor you know, so that's the difference sort of thing.

(Interview 3)

Thus there was no perception of an overlap between the roles of the nurse practitioner and the practice nurse and, interestingly, other nurses were not mentioned, with the exception of the health visitor.

\section{Health visitors}

There was no noticeable role overlap reported between health visitors and the nurse practitioner. One health visitor said: 'We have very little professional contact with the nurse practitioner. Our roles don't overlap at all'. However, there was a perception of different skills, which it was felt that the nurse practitioner's role encompassed: 'Exam- ination skills, referral skills, knowledge of how society can affect health. We just identify need, we can't diagnose'.

However, the health visitors were clear about their own 'turf' and expected the nurse practitioner to refer to them children with problems relating to feeding, potty training or sleep. They were willing for the nurse practitioner to deal with rashes and minor ailments.

A change in the involvement of health visitors at the practice occurred after the introduction of the nurse practitioner. At the doctors' request the health visitors began operating an open-access clinic for families each afternoon. This clinic, which deals mainly with common complaints in the under-fives, receives patients with undiagnosed, undifferentiated conditions, and the health visitors either assess and manage them themselves, or refer them to the doctor on duty. Thus the health visitors are undertaking a triage role similar to that of the nurse practitioner.

The clinics are 'covered' by a GP, but the health visitors felt that the nurse practitioner "would be an equally appropriate person to refer on to with some of the more straightforward ailments children are presenting to us with'.

The health visitors said that they were referring to the nurse practitioner some patients who needed to be seen quickly (e.g., a woman with postnatal depression), because it was a way of getting someone seen by a doctor more quickly. In this situation the nurse practitioner is acting as a gatekeeper to the GP and is making the work of other nurses easier.

\section{Midwives}

The nurse practitioner was not a qualified midwife, and therefore was not expected to undertake such duties as fall within the sphere of competence of the qualified midwife. The system of supervision of midwives operates to ensure that only midwives who have notified the relevant authority of their intention to practise are allowed to undertake certain duties related to the care of the pregnant or postpartum woman.

There's not really any difference for us. She's not involved in the midwifery side. She has referred two pregnant women to us, and we see her most days at the surgery.

(Midwife) 


\section{District nurses}

District nurses perceived that their work had not been much affected by the nurse practitioner, and there was no duplication or overlap of work. One district nurse explained:

There's no effect on my role at the moment. She might refer someone to us - say a leg ulcer. She might do home visits to check drugs if we're not going in. I think there's overlap between primary health care team members.

The district nurses, like the other nurses who were interviewed, perceived the nurse practitioner's role in terms of physical assessment, diagnosis and treatment, and did not regard this as being part of their own role at the present time. Research skills were also mentioned. One district nurse described the skills of the nurse practitioner as being different to those of other nurses in terms of 'diagnostic skills, health promotion [and] research skills'. A district nurse said that the nurse practitioner role was moving nursing forward:

I think it's the way that nursing is going. There's a lot of nurse practitioner jobs advertised. I think we're very capable to work as junior doctors; I think it's your nursing background that gives you such a good background to do that job.

Another district nurse said that the GPs had suggested that district nurses (not the nurse practitioner) could undertake the home visits to patients who had telephoned for a home visit from a GP:

to assess them and see if there's a need for the doctor to go. The GPs are quite keen about her [nurse practitioner] role and what she's able to do. I think they'd actually like to see more nurses doing more.

(District nurse)

This statement was supported by a GP who explained nurses' expanded roles in relation to wider changes in nursing:

I don't know how much of it is a reflection of what's going on in the outside world as what's happening internally. It [the nurse practitioner role] has certainly shown the partners that nurses can take on an increased role in assessment of patients, and the practice is now moving forward in getting the other nurses to potentially triage patients at particular times.

(GP)

There was reported raised awareness among nurses in the practice that they could take on more in relation to patient care:

I mean a number of nurses have expressed this: that the nurse practitioner post is showing them that when a doctor is called in then normally they just switch off - it's now the doctor's job to examine patients, take a history again, and come to a conclusion. Whereas a nurse practitioner is, and the idea behind a nurse practitioner, is showing them that they ought to take an interest in that now. That their role does not stop at assessing the need to see a doctor, and their role can be extended to examining, taking a history, coming to a conclusion, and maybe getting a doctor to confirm or offer a different diagnosis.

(Nurse practitioner)

\section{General practitioners}

The nurses who were interviewed were not clear about where the role of the nurse practitioner ended and that of the doctor began. Some saw it as a continuum, including a midwife who said: 'It's a sort of halfway point really - more than a nurse, but not a doctor - a specialist nurse' (midwife). A district nurse thought that 'the nurse practitioner role is a bit of nursing and a bit of medical. More aligned to GPs - no hands-on nursing'. This perception that there is a blurring of roles between doctors and nurses was regarded by one GP as inevitable and acceptable:

There's a blurring of roles of doctors and nurses as specialist skills increase. Properly so. There are no major disadvantages, but we all have to be certain that the nurse practitioner does acquire skills that a GP has. There are no more objections from patients seeing the nurse practitioner than we would get from a new partner - less actually, which 
is interesting. It's more what the partners do rather than the practice nurse's role.

(GP)

The views of the doctors and nurses reflect several aspects of the nurse practitioner's role, such as diagnostic skills, clinical skills, and skills related to examining, health promotion, extra knowledge, observation, referral, responsibility, accountability, identifying signs and symptoms, listening, counselling and competence. The acquisition of these skills was seen to mark the difference between nurse practitioners and other nurses. For example:

I think she's a different being, she really is. I mean she may not like it but she is becoming very much more a medical role. That's because of her experience. She is probably taking on far more responsibility than other nurses opposite her - it's experience.

(GP)

Some GPs placed limits on the role:

There are obviously limits that have to be drawn. This must be negotiated as the role evolves and people learn to trust each other nurse and doctor. At the end of the day, can she recognize a problem in a patient who needs to be treated, or can she manage herself?

Other GPs saw a complete blurring of the work of the GP and the nurse practitioner, and considered that the nursing aspects of the role are important:

They should hang on to it firmly as a nursing role to deal with, you know, common clinical problems really. It's just as valid an approach, and I mean the medical and nursing approaches are merging into each other anyway ... we're not out there to cure everything. We know we can't do that. I think we're all into a more care approach - a nursing care plan approach which is of more benefit to a lot of problems.

Audits of the nurse practitioner's work showed that by the end of the pilot period she managed $94 \%$ of patients without referral to a doctor. There were few referrals to nurses and other agencies. She was seeing patients with a wide range of conditions, many of which were said to be 'minor', but nevertheless representing much of the normal range of conditions that are seen in general practice. In this regard, the boundary lines between the work of the nurse practitioner and that of the GPs are indeed being redrawn. This accords with the findings of other UK research on the nurse practitioner in general practice (Stilwell et al., 1987; Salisbury and Tettersell, 1988; Marsh and Dawes, 1995).

\section{Patients' perceptions of GP and nurse practitioner roles}

Patients perceived that there was considerable role overlap with GPs, and sometimes there was ambiguity about the nurse practitioner's role in relation to that of GPs. In general, she was seen to be 'like a doctor but not a doctor'. A few patients thought that she was less well qualified than a doctor, and some thought that she was a locum or a trainee.

I thought she was sort of, you know, in between a nurse and a doctor which, you know, it probably is that sort of thing. She seems to know everything, and the only difference is she got the prescription signed by the doctor. She's more a family person, more on a nursing level. I just felt as if I'd been treated by a doctor. It didn't bother me that she wasn't a real doctor. She's more than a nurse I think, more like a doctor. She just filled forms out like a doctor and I don't think nurses can give treatment out like doctors do and she did. But it's just how it came across. Why should there be a nurse practitioner and not another doctor?

(Interview 12)

Certain structures and processes that operate in a symbolic manner provide cues for patients as to how the doctors' and nurses' roles are enacted. These include uniforms, physical space, forms of address, equipment used, and so on. However, the nurse practitioner's role is a departure from the traditional image of a nurse, and symbols such as a room of her own (which differentiates her from the practice nurse) and a desk rather than a trolley or couch in the treatment room provide cues which 
may be confusing for patients. Another departure is the nature of the consultation - for example, the way in which 'she asks questions' (Interview 3), and 'She looks a bit like a doctor' (Interview 5). The manner in which other practice staff referred to or behaved towards the nurse practitioner was another factor. As one patient explained:

Nobody made any difference between them. When you go in to her you treat her exactly like a doctor, so it was obvious she can do exactly what a doctor can do.

(Interview 11)

Several patients did not know the difference between a nurse practitioner and a doctor prior to the consultation, and there was some evidence that several still did not know the difference when they came out of the consulting-room. This has implications for publicizing the role more widely, and it may also have legal implications (Dowling et al., 1996).

Differences between consultations with doctors and consultations with the nurse practitioner were apparent in many patients' perceptions. These differences included not feeling rushed, and the nurse practitioner listening, giving full explanations and being easy to talk to. Clinical competence was highly valued. Some patients felt that doctors would benefit from the nurse practitioner role, as it would take the pressure off them, and there was a recognition that not all consultations need to be with a doctor. Some problems can be dealt with by the nurse practitioner, thereby freeing up the doctor's time for more serious problems requiring special skills, or requiring hospitalization, or 'death-defying things' and 'lumps'.

\section{Discussion}

The implementation of the nurse practitioner role does not appear to increase the work of other nurses noticeably. The only work that had passed to other members of the nursing team from the nurse practitioner consisted of 'a few' patients to the practice nurses, two patients to the health visitors and two patients to the midwives. The reason is likely to be that the nurse practitioner is seeing many patients with minor, self-limiting conditions that do not require follow-up. Those patients who require further investigation, treatment or followup are transferred to the GPs. Therefore referrals for practice nursing, health visiting and district nursing services are more likely to come from the doctors. The nurse practitioner largely undertakes her own procedures, with the exception of administering injections. The traditional nursing skills of the nurse practitioner are being used, as well as the newly acquired skills of physical assessment and diagnosis. Patients perceived practice nurses as being busy, with less time to spend with patients than the nurse practitioner, and they considered that they were task oriented. It was the practice nurse's job to take bloods, give injections, remove stitches and do general health checks.

With regard to midwives there was almost no blurring of roles, as the midwives perceived their role to be very different to that of the nurse practitioner. However, this raises difficulties, as the boundaries are not clear-cut. For example, is testing the urine of a pregnant woman always the responsibility of a midwife? Is not a qualified general nurse qualified to test urine and act on the findings?

There was seen to be considerable overlap with the work of the GPs with regard to consulting with 'urgent' patients. Nurses and patients saw a difference between nurse practitioners and doctors which was mainly related to consultation style, accessibility and length of consultation. Audits of the nurse practitioner's work showed that she was relieving the doctors of many of the 'urgent' patients with 'minor conditions', as was found in several UK studies (Stilwell et al., 1987; Salisbury and Tettersell, 1988; Marsh and Dawes, 1995).

There was little reported role conflict between team members with regard to the nurse practitioner role, and in this respect this study does not support the literature, which warns that team conflict and turbulence are likely to occur when a nurse practitioner is introduced into the team (Stilwell, 1988; Kaufman, 1996). The lack of conflict may be due to several factors, but Abbott (1988) argues that in the face of competition from other professional groups, professions have to protect and maintain the boundaries of their work. Whether consciously or unconsciously, the following boundary maintenance strategies were in place in the case-study practice. 
Aligning the nurse practitioner with the GP rather than the nursing staff

This seemed to reduce the threat to existing nursing roles, as the nurses did not perceive their established roles as being eroded. However, aligning the nurse practitioner with the GP reinforces the medical rather than the nursing aspects of patient care, and because technical skills are emphasized, it may give rise to the nurse practitioner being regarded as a 'mini-doctor' and cause confusion among patients. Therefore both the nurse practitioner and the reception staff need to continually inform patients of her nursing role.

\section{Using the nurse practitioner as a 'back-door' to the GP}

This sometimes involved nurses asking the nurse practitioner for a 'second opinion' about a patient, or referring patients to her knowing that they would be seen sooner than if an appointment was requested with a GP. In this way the nurse practitioner was viewed as an asset rather than a threat.

Emphasizing the differences in skills between the different nursing specialities

This enabled nurses to maintain their specialist focus and sense of professional identity. Provided that the nurse practitioner did not cross the boundary line into health visiting, midwifery, district nursing or practice nursing, and provided that she made 'appropriate' referrals to these professionals, boundary lines could be maintained.

\section{Limitations of the study}

The findings of the interviews with patients indicate a high level of satisfaction with the service provided by the nurse practitioner. This is not surprising, as the patients who agreed to be interviewed had indicated on their completed questionnaires that their encounter with the nurse practitioner had been satisfactory. However, the aim of this part of the study was to find out how they perceived this role in relation to other professionals within the practice. Another limitation is that the responses of male and female patients were analysed together as if they were a homogenous group. However, analysis of sex differences in consumer perceptions was not the aim of the study, although it would be an interesting piece of research in its own right. Furthermore, the responses of children under 16 years of age will almost certainly reflect the views of their carers. In fact this is not too important, as the carers are consumers of the service on behalf of their dependants, so their views are very significant.

The membership of the primary health care team changed over the period of the study, and as new team members arrived they had to get used to working with a nurse practitioner. This is one of the problems of a longitudinal study, as no one can predict that everything will remain constant over time. This survey only provides a snapshot in time, and as the role of the nurse practitioner becomes more firmly established within the practice, perceptions may change.

\section{Conclusion}

Several key themes emerged from this study.

First, with regard to role boundaries, the nurses in the practice and those working in the community did not experience any infringement of their own roles and there was little overlap. Where referrals were made from the nurse practitioner to other nurses, these were thought to be appropriate. There was a clear division of labour among members of the nursing team at the time of the study, which helped to reduce conflict, as everyone knew their own sphere of practice, and although there was sharing of work, there was little dissent. The nurse practitioner was perceived as a 'different' type of nurse - one who had extra skills and knowledge and who worked closely with the GPs.

Secondly, there was much overlap with the work of the GPs, but this reflects the nature of the role as defined in this particular practice. It is a very similar role to that of many nurse practitioners working in general practice.

Thirdly, patients perceived a clear difference between nurse practitioners and practice nurses. The practice nurses were perceived as being concerned with technical aspects of care, whilst the nurse practitioner was regarded as having a wider remit, closer to that of the doctor. Doctors and nurses supported this perception. However, care must be taken to publicize the role well in order to avoid confusion among patients.

The nurse practitioner in this particular practice was a very experienced practice nurse with 
a wide range of knowledge and skills which she brought to the role. This, together with wellorganized teaching and supervision in the practice setting, and a structured programme of formal education, resulted in a highly effective nurse practitioner who was generally perceived to have blurred the boundary lines between medical and nursing roles.

\section{References}

Abbott, A. 1988: The system of professions: an essay on the division of expert labour. Chicago: University of Chicago Press.

Burke-Masters, B. 1986: The autonomous nurse practitioner: an answer to a chronic problem of primary care. Lancet 1, 1266.

Cable, S. 1994: What is a nurse practitioner? Primary Health Care 4, 12-14.

Department of Health and Social Security 1986: Neighbourhood nursing - a focus for care. Report of the Community Nursing Review (the Cumberlege Report). London: HMSO.

Dickson, N. 1996: Are nurse practitioners merely substitute doctors? Professional Nurse 11, 326.

Dowling, S., Martin, R., Skidmore, P., Doyal, L., Cameron, A. and Lloyd, S. 1996: Nurses taking on junior doctors' work: a confusion of accountability. British Medical Journal 312, 1211-14.

Emmerson, P. 1996: Are nurse practitioners merely substitute doctors? Professional Nurse 11, 326.

Greenfield, S., Stilwell, B. and Drury, M. 1987: Practice nurses: social and occupational characteristics. Journal of the Royal College of General Practitioners 37, 341-45.

Hupcey, J. E. 1993: Factors and work settings that may influence nurse practitioner practice. Nursing Outlook 41, 181-85.

Jenkins-Clarke, S., Carr-Hill, R., Dixon, P. and Pringle, M.
1997: Skill mix in primary care: a study of the interface between general practitioners and other members of the primary health care team. York: University of York Centre for Health Economics.

Kaufman, G. 1996: Nurse practitioners in general practice: an expanding role. Nursing Standard 11, 44-47.

MacGuire, J. 1980: The extended role of the nurse. London: King's Fund Centre.

Marsh, G.N. and Dawes, M.L. 1995: Establishing a minor illness nurse in a busy general practice. British Medical Journal 310, $778-80$.

Molde, S. and Diers, D. 1985: Nurse practitioner research: selected literature review and research agenda. Nursing Research 34, 362-66.

Oakley, A. 1984: The importance of being a nurse. Nursing Times 80, 24-27.

Pearson, P. 1996: Are nurse practitioners merely substitute doctors? Professional Nurse 11, 325.

Poulton, B. 1995: Keeping the customer satisfied. Primary Health 5, 16-19.

Reveley, S. 1999: Introducing the nurse practitioner into general medical practice: operational and theoretical perspectives on the role. Unpublished $\mathrm{PhD}$ thesis. Lancaster: University of Lancaster.

Salisbury, C.J. and Tettersell, M. 1988: Comparison of the work of a nurse practitioner with that of a general practitioner. Journal of the Royal College of General Practitioners 38, 314-16.

Stilwell, B. 1988: Patient attitudes to a highly developed role the nurse practitioner. Recent Advances in Nursing 12, 82-100.

Stilwell, B., Greenfield, S., Drury, V.W.M. and Hull, F.M. 1987: A nurse practitioner in general practice: working style and pattern of consultations. Journal of the Royal College of General Practitioners 37, 154-57.

Witz, A. 1994: The challenge of nursing. In Gabe, J., Kelleher, D. and Williams, G., (editors). Challenging medicine. London: Routledge, 23-45. 